\title{
Intrauterine hyperglycemia exposure results in intergenerational inheritance via DNA methylation reprogramming on F1 PGCs
}

Jun Ren ${ }^{1,2+}$, Yi Cheng ${ }^{1,2+}$, Zhen-Hua Ming ${ }^{2,3}$, Xin-Yan Dong 1,2 , Yu-Zhong Zhou 1,2, Guo-Lian Ding ${ }^{4}$, Hai-Yan Pang ${ }^{1,2}$, Tanzil Ur Rahman ${ }^{1,2}$, Rubab Akbar ${ }^{1,2}$, He-Feng Huang ${ }^{2,4^{*}}$ and Jian-Zhong Sheng ${ }^{1,2^{*}}$

\begin{abstract}
Background: The existing reports about intergenerational or transgenerational effects of intrauterine hyperglycemia have included both intrauterine and postnatal metabolic exposure factors, while the impact of intrauterine hyperglycemia per se has not been assessed alone. A number of studies suggest DNA methylation reprogramming of gametes plays a crucial role in the metabolic inheritance, but it is unclear when and how DNA methylation patterns are altered when exposed to intrauterine hyperglycemia. In this study, we selected nondiabetic F1- and F2-gestational diabetes mellitus (GDM) male mice as founders to examine metabolic changes in the next generation and performed methylome sequencing of day 13.5 primordial germ cells (PGCs) from F1-GDM to explore the underlying epigenetic mechanism.
\end{abstract}

Results: We found that intrauterine hyperglycemia exposure resulted in obesity, insulin resistance, and/or glucose intolerance in F2 male mice, but no metabolic changes in F3 male mice at 8 weeks. Using reduced representation bisulfite sequencing, we found DNA methylome of day 13.5 PGCs from F1-GDM fetuses revealed differently methylated genes enriched in obesity and diabetes. Methylation validation of the insulin resistance and fat accumulation gene Fyn showed a consistent hypomethylation status in F1 PGCs, F1 fetal testes, sperm from F1/C-GDM mice, and somatic cells from F2-GDM male mice. In contrast, no methylation alteration was observed in F2-GDM male germ cells and F3-GDM somatic cells.

Conclusion: We provide evidence that intrauterine hyperglycemia exposure per se contributes to intergenerational metabolic changes in the F2 but not F3 generation. And the aberrant DNA methylation reprogramming occurs as early as day 13.5 in PGCs of the F1 generation. Our findings suggest that intrauterine exposure alone is sufficient to cause the epigenetic inheritance in F2 offspring, and the epigenetic memory carried by DNA methylation pattern could be erased by the second wave of methylation reprogramming in F2 PGCs during fetal development.

Keywords: DNA methylation, Intrauterine hyperglycemia, Primordial germ cells, Reduced representation bisulfite sequencing, Epigenetic inheritance

\footnotetext{
*Correspondence: huanghefg@hotmail.com; shengjz@zju.edu.cn

†Jun Ren and Yi Cheng contributed equally to this work.

${ }^{1}$ Department of Pathology and Pathophysiology, School of Medicine,

Zhejiang University, Hangzhou, China

${ }^{2}$ The Key Laboratory of Reproductive Genetics (Zhejiang University)،

Ministry of Education, Hangzhou, China

Full list of author information is available at the end of the article
} 


\section{Background}

The concept of "developmental origins of health and disease $(\mathrm{DOHaD})$ ", which states that an adverse intrauterine environment during critical periods in early life may lead to disease risk in later life, is now widely accepted [1]. This theory has been supported by abundant results from both human epidemiological studies and animal models [2-6]. Adverse factors, such as imbalanced nutrition intake, smoking, caffeine, bisphenol A, and drug use, affect multiple aspects of offspring health [2-6]. Accumulating evidence also suggests that adverse intrauterine exposure may not only directly influence the development of the fetus (F1 offspring), but also the germ cells of F1 that will form the F2 generation [7].

Gestational diabetes mellitus (GDM) is defined as glucose intolerance and hyperglycemia with first onset during pregnancy [8]. GDM is the most common complication of pregnancy, affecting $5-10 \%$ of all pregnancies [9]. Children born to mothers with GDM are prone to develop chronic diseases such as obesity, impaired glucose tolerance, cardiovascular disorders, and type II diabetes in adulthood [10-13]. In addition, our previous study suggested that the second generation might exhibit similar metabolic dysfunction phenotypes to their parents, especially by paternal line [14]; however, in this study sperm of the F1 generation underwent two major phases of adverse environment exposure: prenatal exposure to intrauterine hyperglycemia and postnatal metabolic dysfunction to the F1 generation itself. It has been demonstrated that postnatal induced-diabetic conditions in healthy male mice could alter the epigenetic information in sperm, and these epigenetic changes were largely heritable [15]. The offspring showed diabetic phenotypes that resembled their fathers [15]. Up to now, it is unclear whether intrauterine hyperglycemia exposure itself may affect epigenome of gamete precursors of the F1 fetus. Therefore, it is necessary to investigate the effects of intrauterine hyperglycemia on epigenetic alteration in primordial germ cells (PGCs) of the F1 fetus and assess whether and how intrauterine hyperglycemia results in metabolic changes in the F2 generation.

The term transgenerational inheritance is broadly used to describe all nonsequence-based effects that can be transmitted to next generation. However, it is important to distinguish transgenerational effects from intergenerational effects. Truly transgenerational effect is found in generations that were not exposed to the initial environmental exposure that trigger the change. A nexus of mechanisms may contribute to the transmission effect, including self-sustaining feedback loops, chromatinbased mechanisms, noncoding and coding RNA, even nonepigenetic mechanisms like microbiotic effects or metabolites [16]. DNA methylation is the best studied chromatin-based mechanism in this field.

DNA methylation is a major form of epigenetic modification; it is crucial for the development and differentiation of various cell types in an organism. Substantial evidence has demonstrated that altering the DNA methylation pattern contributes to the inheritance of acquired characteristics [17-20]. Two waves of genome-wide DNA methylation reprogramming occur during mouse development. The first wave of reprogramming occurs in pre-implantation blastocyst embryos, and the second wave occurs solely in PGCs [21]. PGCs are specified in mice during gastrulation. The second wave of global DNA methylation modification of PGCs is erased at day (D) 12.5-13.5 in mice after colonization into the genital ridges $[22,23]$ and then re-established when PGCs differentiate into the precursors of gametes at D18.5 [24].

Previous studies that investigated the mechanisms underlying the intergenerational effects of GDM have usually focused on mature germ cells rather than PGCs. Therefore, it has not yet been studied whether exposure to intrauterine hyperglycemia affects the DNA methylation reprogramming of PGCs. In this study, we built a GDM mouse model and selected male F1- and F2-GDM mice without the pre-diabetic phenotype as founders to produce the next generation. Thereby we investigated the direct effects of intrauterine hyperglycemia exposure on the F2 and F3 generations. We also assessed DNA methylation reprogramming in male PGCs from F1 fetuses, and its heritability to germ cells and somatic cells in F2 and F3 generation.

\section{Methods}

\section{Establishing the GDM mouse model}

The protocol was approved by the Zhejiang University Institutional Animal Care and Use Committee. The animals were housed in a temperature- and humiditycontrolled environment under a 12-h light/dark cycle. Eight-week-old normal virgin female ICR mice were mated with normal males overnight. The onset of pregnancy was determined by the presence of a vaginal plug the following morning and defined as D0.5 of gestation. Gestational mice (F0) were randomly divided into control and intrauterine hyperglycemia (GDM) groups. After an 8-h fast, mice in the GDM group were treated with a single intraperitoneal injection of streptozotocin (STZ; Sigma, St. Louis, MO, USA) in $0.1 \mathrm{mM}$ citrate buffer (pH 4.5) at a dose of $150 \mathrm{mg} / \mathrm{kg}$ bodyweight. Mice in the control group received an equal volume of citrate buffer. On D3.5 of pregnancy, the blood glucose concentration was measured via the tail vein using a glucometer (Roche Diagnostics Accu-Chek, Mannheim, Germany). Diabetes was defined as a glucose level between 14 and $19 \mathrm{mM}$ [25, 
26]. Blood glucose levels were also monitored on D7.5 of pregnancy to confirm the presence of diabetes. The pregnant mice were allowed to deliver spontaneously. Pups born to the GDM mother (F1-GDM) were fostered by normoglycemic control mothers until they were weaned at 3 weeks of age. All mice were fed a standard diet.

F1-GDM male mice without metabolic phenotypes were collected as F1/C-GDM. F1/C-GDM mice were mated with control females to produce F2-GDM. Similarly, F2-GDM male mice without metabolic phenotypes (F2/C-GDM) were mated with control females to produce F3-GDM. In our experiments, one GDM mother was capable of producing both GDM and C/GDM offspring. Only male pups were investigated.

Glucose tolerance test (GTT) and insulin tolerance test (ITT) For GTT, animals were fasted for $12 \mathrm{~h}$ before the test. An intraperitoneal injection of glucose ( $2 \mathrm{~g} / \mathrm{kg}$ body weight) was given to each animal, and glucose levels were measured at $0,30,60$, and $120 \mathrm{~min}$ after injection. The area under the curve (AUC) for the GTT was calculated. For ITT, an intraperitoneal injection of insulin $(0.8 \mathrm{U} / \mathrm{kg}$ body weight) was performed after a 3-h fast. Blood glucose concentrations were measured from the tail vein at $0,30,60,90$, and 120 min after injection. The AUC was also calculated for the ITT.

\section{Serum biochemical measurements}

Blood samples were collected from 8-week-old mice after a 12-h fast. The serum levels of fasting insulin were determined with the mouse insulin enzyme-linked immunosorbent assay (ELISA) kit (Crystal Chem, Downers Grove, IL, USA). Serum triglyceride (TG), total cholesterol (TC), high-density lipoprotein (HDL), and low-density lipoprotein (LDL) were assayed using a biochemical analyzer (TBA120FR, Toshiba, Tokyo, Japan). Fasting glucose levels were measured using a glucometer (Roche Diagnostics Accu-Chek). The Homeostasis Model of Insulin Resistance (HOMA-IR) index was calculated using the formula: HOMA-IR $=($ fasting insulin $\times$ fasting glucose)/22.5.

\section{Reduced representation bisulfite sequencing (RRBS) and data analysis}

RRBS (Genergy Biotechnology Co., Ltd., Shanghai, China) was performed in PGCs of D13.5 male fetuses from control pregnant mice (F0-control, $n=8$ ) and GDM pregnant mice (F0-GDM, $n=8$ ). PGCs were pooled from two pregnant mice to obtain the amount of material needed for RRBS. Briefly, $5 \mu$ g genomic DNA was digested using the methylation-insensitive restriction enzyme MspI (New England Biolabs, Beverly, MA, USA). A Qiagen Mini Purification kit (Qiagen, Hilden,
Germany) was used to purify the digested products. Then, the ends of each restriction fragment were filled in and adenosine was added at the $3^{\prime}$-end. Methylated paired-end Illumina adapters were ligated to the ends of the DNA fragments using T4 DNA ligase, and fragments sized 100-200 bp were purified by agarose gel extraction. The purified fragments were treated with sodium bisulfite and then amplified by PCR. The final PCR products were sequenced on HiSeq 2500 (Illumina Inc., San Diego, CA, USA). Differentially methylated loci (DML) and differentially methylated regions (DMRs) were analyzed based on a Bayesian approach [27], summarized as follows: two groups were modeled according to the Bayesian stratification model, and the Wald test was applied to each locus to get a $\mathrm{p}$ value for each $\mathrm{CpG}$ site. For each CpG site, a difference in methylation value between two groups $\geq 5 \%$ and a posteriori probability of Wald test $\geq 0.95$ was considered to be a DML. A methylation region was defined as a DMR when it met these three criteria: (1) the length of this region was at least $50 \mathrm{bp}$; (2) the region contained no less than three CpG sites; (3) the proportion of DMLs in this region was no less than $50 \%$. When a DMR showed no less than $50 \%$ overlap with one element of the gene, it was defined as a differentially methylated gene (DMG). Bioinformatic analysis of DMGs was performed using the Ingenuity Pathway Analysis (IPA) software package (Ingenuity Systems, Redwood City, CA, USA). The RRBS data reported in this paper have been deposited in the NCBI Gene Expression Omnibus (GEO) database with accession number GSE108319.

\section{DNA extraction and pyrosequencing PCR}

DNA was isolated using TIANamp Genomic DNA Kit (Tiangen Biotech, Beijing, China). DNA bisulfite conversion was modified using EZ DNA CT conversion reagent (Zymo Research Corporation, Irvine, CA, USA) following the manufacturer's protocol. The primers for Fyn bisulfite DNA PCR and sequencing were designed using Pyromark Assay Design 2.0 software (Biotage, Uppsala, Sweden) and listed in Additional file 1. Gene model of Fyn was presented as Additional file 1: Fig. S1.

Bisulfite DNA PCR was performed in a total volume of $25 \mu \mathrm{l}$ using the TaKaRa EpiTaq HS system (TaKaRa, Dalian, China). Pyrosequencing was performed on a PyroMark Q24 MD system (Qiagen) using the PyroMark Gold Q24 reagent kit (Qiagen) with 10 pmol of sequencing primer. Data were analyzed using Pyro Q-CpG software (Qiagen).

\section{RNA isolation and quantitative real time-PCR (qPCR) analysis}

RNA was extracted using TRIzol reagent (Life Technologies, Grand Island, NY, USA) and cDNA was synthesized 
using oligo-dT and random primers (TaKaRa). qPCR was performed using the ABI Prism $7900 \mathrm{HT}$ sequence detection system (Applied Biosystems, Foster City, CA, USA) with commercial primers generated for the system. $\beta$-actin was used as the internal control. Primers were listed in Additional file 1.

\section{Statistical analysis}

SPSS Statistics 16 (SPSS Inc., Chicago, IL, USA) was used for all statistical analyses. Data are presented as mean \pm SEM. Comparisons between two groups were performed using Student's $t$ tests. One-way ANOVA was used to detect differences among multiple groups followed by LSD post hoc test for comparing groups. All reported $p$ values are two-sided. $p<0.05$ was considered statistically significant.

\section{Results}

F1/C-GDM male mice had normal glucose and lipid metabolism

GTT is the most commonly used method for assessing glucose homeostasis, and it provides a physiological overview of any changes in glucose tolerance. ITT and HOMA-IR index are used to measure insulin resistance of peripheral tissues. These three methods along with basal hormone levels provide a systemic view of glucose homeostasis. GTT and ITT tests showed that, compared with control male mice, 8-week-old F1-GDM male mice exhibited significant glucose intolerance (Fig. 1a, b). Though F1-GDM mice showed a significantly higher glucose level at $120 \mathrm{~min}$ in ITT, there was no difference in AUC of ITT (Fig. 1d, e) or HOMA-IR index (Fig. 1f) between the two groups. Further analysis showed that $36 \%(9 / 25)$ of the F1-GDM male offspring had normal glucose tolerance and insulin tolerance (Fig. 1b, e). These mice were separated and defined as GDM offspring without impaired glucose tolerance and impaired insulin tolerance (F1/C-GDM). There were no significant differences in bodyweight (Fig. 1c), fasting glucose level, fasting insulin level, TG, TC, HDL, LDL (Table 1), and HOMA-IR index (Fig. 1f) between the control and F1/CGDM groups.

Adult F2-GDM male mice from F1/C-GDM fathers exhibited obesity, insulin resistance, and/or glucose intolerance

F1/C-GDM male mice were mated with control female virgin to produce second-generation mice, named F2-GDM. GTT was conducted at 8 weeks. Then, the F2-GDM mice were divided into two groups according to the AUC of the GTT: F2/H-GDM (with impaired glucose tolerance) and F2/N-GDM (with normal glucose tolerance). Glucose levels were significantly higher at 30 and 60 min in F2/H-GDM mice compared with the control group ( $p<0.05$ and $p<0.001$, respectively; Fig. $2 \mathrm{a})$. The AUC of F2/H-GDM mice was significantly larger than that of control and F2/N-GDM mice $(p<0.001$; Fig. $2 \mathrm{~b})$. There was no difference in GTT between the F2/N-GDM and control groups (Fig. 2a, b).

At 8 weeks of age, the mean bodyweight of mice in the $\mathrm{F} 2 / \mathrm{H}-\mathrm{GDM}$ and $\mathrm{F} 2 / \mathrm{N}-\mathrm{GDM}$ groups was significantly higher than that of control mice $(p<0.001$ and $p<0.001$, respectively; Fig. 2c). There was no significant difference in bodyweight between F2/H-GDM and F2/N-GDM mice (Fig. 2c).

ITT was performed to assess insulin sensitivity of the F2 generation. Although glucose levels were only increased in F2/H-GDM mice at $90 \mathrm{~min}(p<0.05)$, the AUC of the ITT was increased significantly $(p<0.05)$ compared with control mice. The F2/N-GDM group exhibited continuously higher glucose levels at 30, 60, 90, and $120 \mathrm{~min}(p<0.01, p<0.001, p<0.001$, and $p<0.001$, respectively), and the AUC was significantly larger than that of the control group $(p<0.001$; Fig. $2 \mathrm{~d}$, e).

HOMA-IR demonstrated the same trend as the ITT results in the F2 generation. There were no differences in fasting glucose levels among the three groups. Fasting insulin was higher in the F2/H-GDM group than the control group, but not significantly (Table 1 ). In the F2/NGDM group, fasting insulin levels were significantly increased compared with the control and F2/H-GDM groups $(p<0.001$ and $p<0.01$, respectively; Table 1$)$. HOMA-IR was significantly higher in the F2/H-GDM group than the control group ( $p<0.05$; Fig. $2 \mathrm{f})$. In addition, HOMA-IR was significantly higher in the F2/NGDM group than the control and F2/H-GDM groups $(p<0.001$ and $p<0.01$, respectively; Fig. $2 \mathrm{f})$. There were no differences in serum TC, TG, HDL, and LDL levels between F2/N-GDM and control mice (Table 1). In contrast, F2/H-GDM mice exhibited elevated TG and reduced LDL levels compared with control $(p<0.05$, and $p<0.05$, respectively; Table 1 ). Finally, TG levels were significantly higher in $\mathrm{F} 2 / \mathrm{H}-\mathrm{GDM}$ mice compared with F2/N-GDM $(p<0.05$; Table 1). Several F2-GDM mice without any abnormal phenotype were selected as F2/CGDM mice to produce F3 generation (Additional file 2: Table S1, Fig. S2). The proportion of F2/C-GDM mice was $15 \%$ (6/40 in F2-GDM male mice), less than that in F1/C-GDM.

The methylome of D13.5 PGCs in F1 male fetus was altered by intrauterine hyperglycemia exposure

In our F1/C-GDM mouse model, exposure to hyperglycemia occurred only during pregnancy with no exposure after birth. However, this exposure resulted in next-generation obesity, insulin resistance, and/or glucose intolerance. This suggests that altered heritable information 

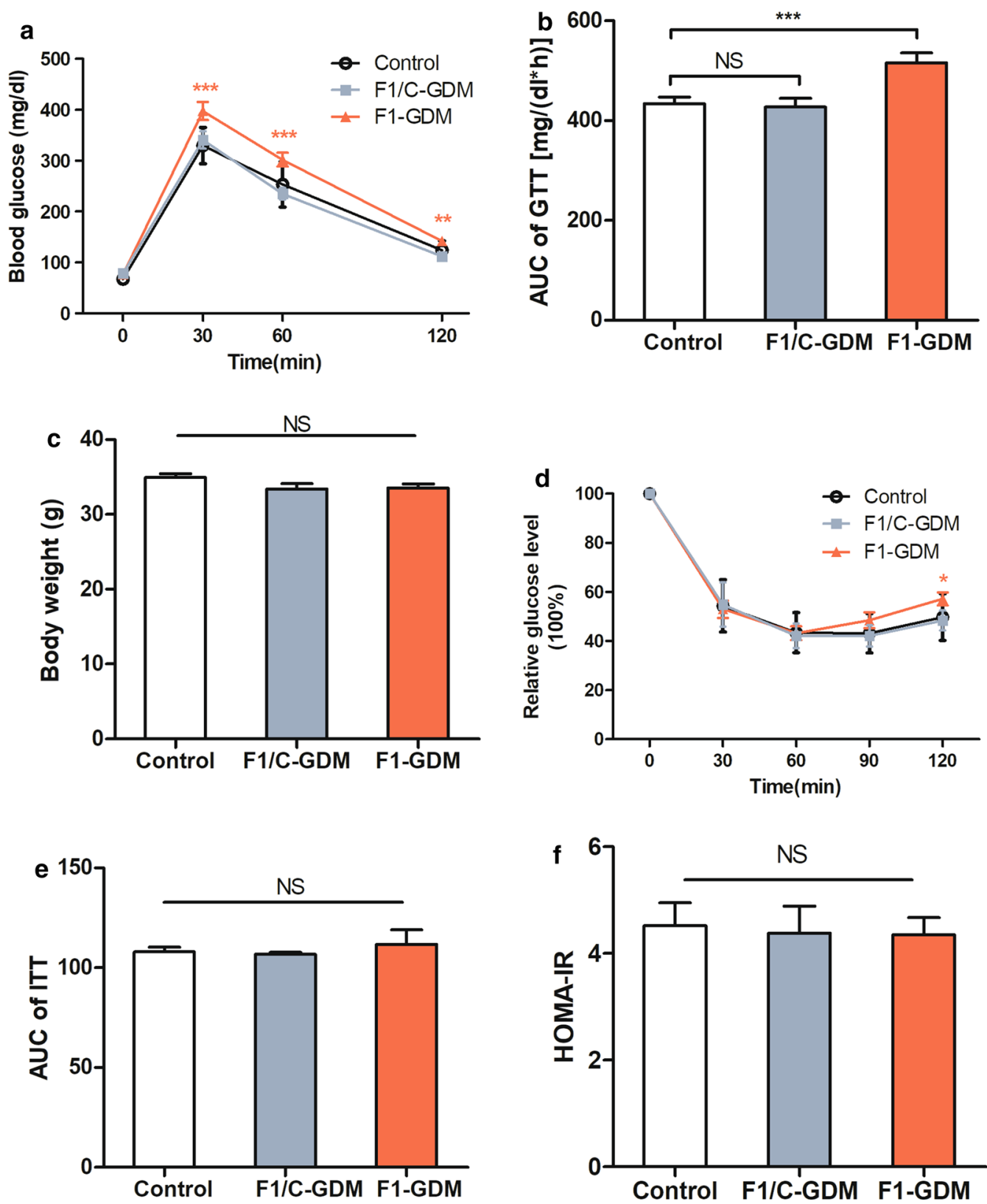

Fig. 1 F1/C-GDM mice demonstrated an unaffected metabolic phenotype at adulthood. a GTT curve and $\mathbf{b}$ the resulting AUC of control, F1-GDM and F1/C-GDM male mice at 8 weeks. c Bodyweight of control, F1-GDM, and F1/C-GDM male mice at 8 weeks. $\mathbf{d}$ ITT curve and $\mathbf{e}$ the resulting AUC of control, F1-GDM, and F1/C-GDM male mice at 8 weeks. For each group in a-e, control $(n=10)$, F1-GDM $(n=25)$, and F1/C-GDM $(n=9)$. f HOMA-IR index of control $(n=10), \mathrm{F} 1-\mathrm{GDM}(n=10)$, and F1/C-GDM $(n=4)$ male mice at 8 weeks. Data are presented as mean \pm SEM. ${ }^{*} p<0.05$, ${ }^{* *} p<0.01,{ }^{* * *} p<0.001$ versus the corresponding control

carried by gametes, such as epigenetic DNA methylation, is responsible for this susceptibility. We therefore used genome methylation sequencing to search for differentially methylated loci between control and F1-GDM fetuses. PGCs undergo global methylation erasure at D13.5 during pregnancy [28], and the methylation status was relatively stable [21]. We hypothesized that early epigenetic reprogramming in PGCs was altered during intrauterine hyperglycemia exposure. To test this hypothesis, we selected D13.5 PGCs from control and GDM fetuses and performed RRBS.

The gender of the D13.5 PGCs was identified under an anatomical lens and confirmed by Sry PCR results (Additional file 1: Fig. S3). Intrauterine hyperglycemia 
Table 1 Serum biochemical parameters in F1/C-, F2-, and F3-GDM male mice at 8 weeks

\begin{tabular}{lccccc}
\hline & Control $(\boldsymbol{n}=\mathbf{1 0})$ & F1/C-GDM $(\boldsymbol{n}=\mathbf{9})$ & F2/H-GDM $(\boldsymbol{n}=\mathbf{1 0})$ & F2/N-GDM $(\boldsymbol{n}=\mathbf{6})$ & F3-GDM $(\boldsymbol{n}=\mathbf{1 0})$ \\
\hline Fasting glucose $(\mathrm{mM})$ & $4.57 \pm 0.23$ & $4.38 \pm 0.24$ & $4.78 \pm 0.20$ & $4.54 \pm 0.02$ & $4.61 \pm 0.31$ \\
Fasting insulin (mIU/l) & $22.05 \pm 6.97$ & $21.87 \pm 4.50$ & $27.81 \pm 9.95^{\# \#}$ & $73.12 \pm 9.45^{* * *}$ & $19.10 \pm 7.43$ \\
TC (mM) & $2.47 \pm 0.06$ & $2.65 \pm 0.11$ & $2.48 \pm 0.11$ & $2.48 \pm 0.09$ & $2.29 \pm 0.16$ \\
TG (mM) & $1.33 \pm 0.11$ & $1.32 \pm 0.17$ & $1.83 \pm 0.12^{*}, \#$ & $1.20 \pm 0.14$ & $1.24 \pm 0.61$ \\
HDL (mM) & $1.39 \pm 0.03$ & $1.54 \pm 0.06$ & $1.43 \pm 0.05$ & $1.37 \pm 0.04$ & $1.60 \pm 0.10$ \\
LDL (mM) & $0.20 \pm 0.01$ & $0.20 \pm 0.01$ & $0.16 \pm 0.01^{*}$ & $0.18 \pm 0.02$ & $0.22 \pm 0.01$ \\
\hline
\end{tabular}

$T C$ total cholesterol; TG triacylglycerol; $H D L$ high-density lipoprotein; $L D L$ low-density lipoprotein. All parameters were measured at 8 weeks of age. Values are expressed as mean \pm SEM

${ }^{*} p<0.05 ;{ }^{* * *} p<0.001$ compared with the control group

${ }^{\#} p<0.05 ;{ }^{\# \#} p<0.01$ compared with the F2/N-GDM group

exposure resulted in 19,679 DML that were distributed in the upstream $2 \mathrm{k}(2.79 \%), 5^{\prime}$-untranslated region $\left(5^{\prime}\right.$ UTR, 1.1\%), coding sequence (CDS, 16.02\%), introns (33.48\%), 3'-UTR (2.29\%), downstream $2 \mathrm{k}(2.7 \%)$, and other elements (45.21\%) of genes (Additional file 3: Fig. S4-S5). The 19,679 DML were associated with 304 genes that were then used in IPA analysis (Additional file 4: Table S2).

IPA analysis identified a cluster of differentially methylated genes that were strongly related to diabetes mellitus, glucose metabolism, lipid metabolism, obesity, and insulin resistance (Fig. 3), including hypermethylated genes such as $A k t 1$, which regulates glucose uptake into muscle and fat cells [29]; Elovl5, which regulates blood glucose [30]; Esr1, which is involved in lipid accumulation and insulin sensitivity [31]; Prkca, which is involved in insulin signaling [32], and hypomethylated genes such as Socs2, which leads to hyperglycemia and glucose intolerance [33]; Fyn, which is essential in fat accumulation and insulin resistance [34]; Park2, which targets to mitochondria and potentiates autophagic vesicle synthesis [35]. Pathway details of the IPA analysis (Table S3) were attached as Additional file 5. Further, we performed targeted bisulfite pyrosequencing of Fyn in additional PGC samples from control and GDM groups and confirmed a hypomethylation status at each detected CpG site and a marked decreasing in the mean methylation level of Fyn in GDM PGCs (Fig. 4a). To examine whether STZ would affect DNA methylation, PGCs of STZ-treated nondiabetic mice were collected, targeted bisulfite pyrosequencing of Fyn of this group showed no difference when comparing with control (Additional file 6: Fig. S6).

These results suggest that intrauterine hyperglycemia exposure leads to aberrant methylation erasure in D13.5 PGCs, and many of the differentially methylated genes were diabetes-related.

\section{Epigenetic alteration in $\mathrm{F} 1$ gametes was inherited by $\mathrm{F} 2$ somatic tissues}

We performed RRBS in D13.5 PGCs, since global DNA methylation undergoes re-establishment at D18.5, methylation validation is necessary to investigate whether altered methylation erasure persists after global DNA methylation re-establishment process in later gametes. D18.5 testes from control and F1-GDM fetuses and sperm samples from the control and F1/C-GDM generation were used to investigate epigenetic maintenance in the F1 gametes. Then, D7.5 fetuses, adult liver, adipose tissue, and skeletal muscle from F2-GDM mice were used to investigate the effects of epigenetic inheritance from F1 to F2 generation. Finally, D13.5 PGCs, D18.5 testes from F2-GDM mice, sperm from F2/C-GDM mice, and D7.5 fetuses from F3-GDM were used to investigate the effects of epigenetic inheritance from F2 to F3 generation.

In these experiments, Fyn was investigated as a target gene. In F1 generation, D18.5 testes from F1-GDM fetuses exhibited decreased overall methylation as well as reduced methylation levels at CpG sites 1, 2, and 3 of Fyn (Fig. 4b). In sperm samples, the F1/C-GDM group had significantly lower methylation levels at all five $\mathrm{CpG}$ sites of Fyn (Fig. 4c). The mean methylation level at these five sites of Fyn was also lower than control group (Fig. 4c). Consistently decreased methylation levels of Fyn in D13.5 PGCs, D18.5 testes, and adult sperm from F1/C-GDM mice indicate the strong impact of intrauterine hyperglycemia exposure on DNA methylation status and suggest that alterations in early germ cells can be maintained in mature gametes in adulthood.

In F2 generation, D7.5 F2-GDM fetuses exhibited a significantly lower methylation level of Fyn compared with the control fetus (Fig. 4d). Based on the different phenotypes in F2 adult individuals, the F2/H-GDM and F2/N-GDM groups were analyzed separately. Liver, adipose, and skeletal muscle tissues were used since 

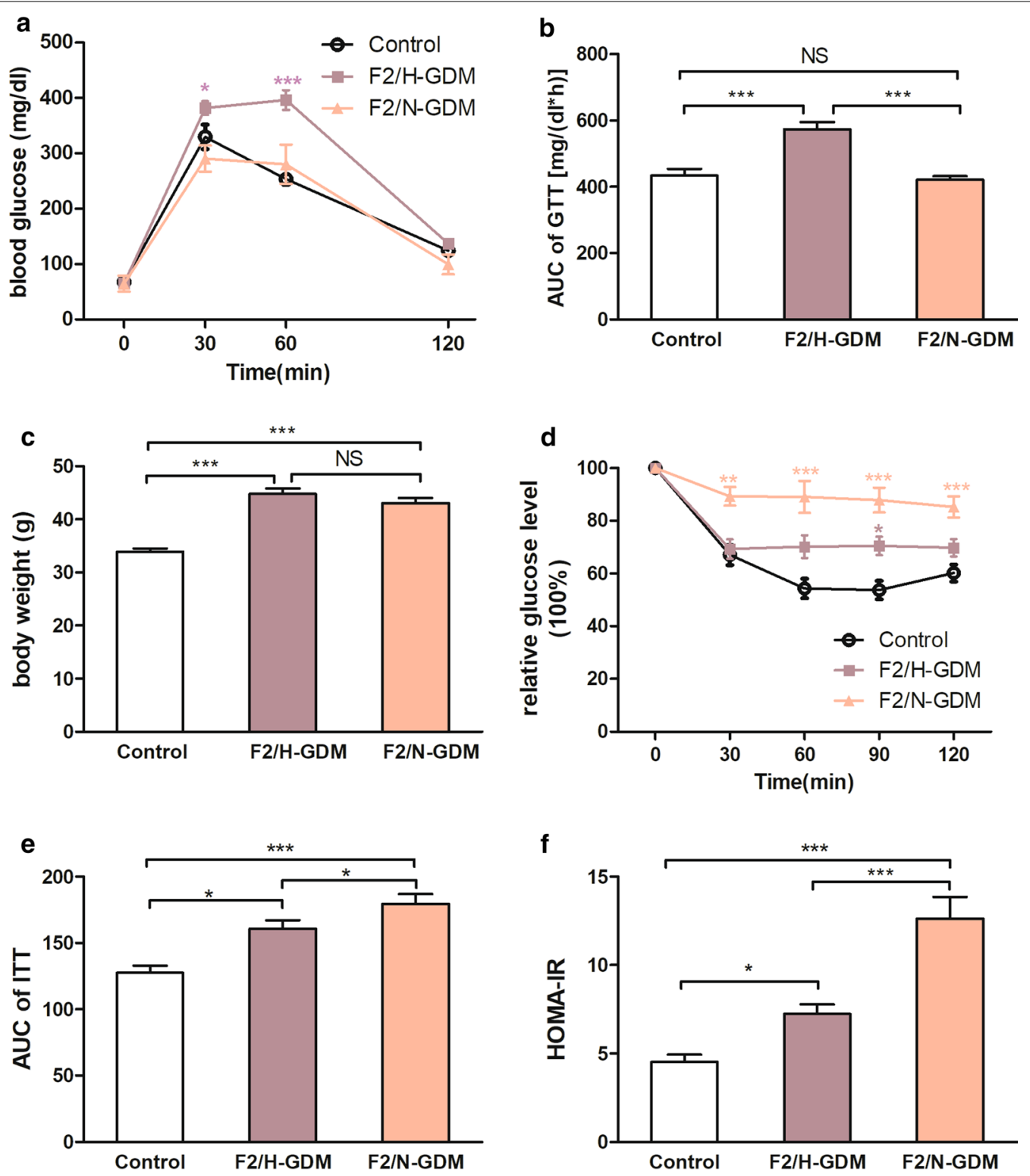

Fig. 2 F2-GDM mice demonstrated an impaired metabolic phenotype at adulthood. a GTT curve and $\mathbf{b}$ the resulting AUC in control, F2/H-GDM, and F2/N-GDM male mice at 8 weeks. c Bodyweight in control, F2/H-GDM, and F2/N-GDM male mice at 8 weeks. $\mathbf{d}$ ITT curve and e the resulting AUC of control, F2/H-GDM, and F2/N-GDM male mice at 8 weeks. For each group in a-e, control $(n=10), F 2 / H-G D M(n=10)$, and F2/N-GDM $(n=6)$. f HOMA-IR index of control $(n=10), \mathrm{F} 2 / \mathrm{H}-\mathrm{GDM}(n=10)$, and F2/N-GDM $(n=6)$ male mice at 8 weeks. Data are presented as mean \pm SEM. ${ }^{*} p<0.05$, ${ }^{* *} p<0.01,{ }^{* * *} p<0.001$ versus the corresponding control

they are the main insulin target tissues. In visceral adipose tissues, F2/H-GDM and F2/N-GDM groups both exhibited significantly lower methylation at single sites and reduced overall methylation of Fyn compared with the control group (Fig. 4e). In addition, qPCR analysis of Fyn in adipose tissues from the F2/H-GDM and F2/N-GDM groups revealed significantly increased expression compared with control (Fig. 5a).
In the liver, the F2/H-GDM group exhibited significant hypomethylation at all five CpG sties (Fig. 4f), and $a>20 \%$ reduced overall methylation rate of Fyn compared with control (Fig. 4f). In addition, the qPCR for Fyn revealed significantly increased expression in the F2/H-GDM group compared with control (Fig. 5b). However, the F2/N-GDM group showed no DNA 


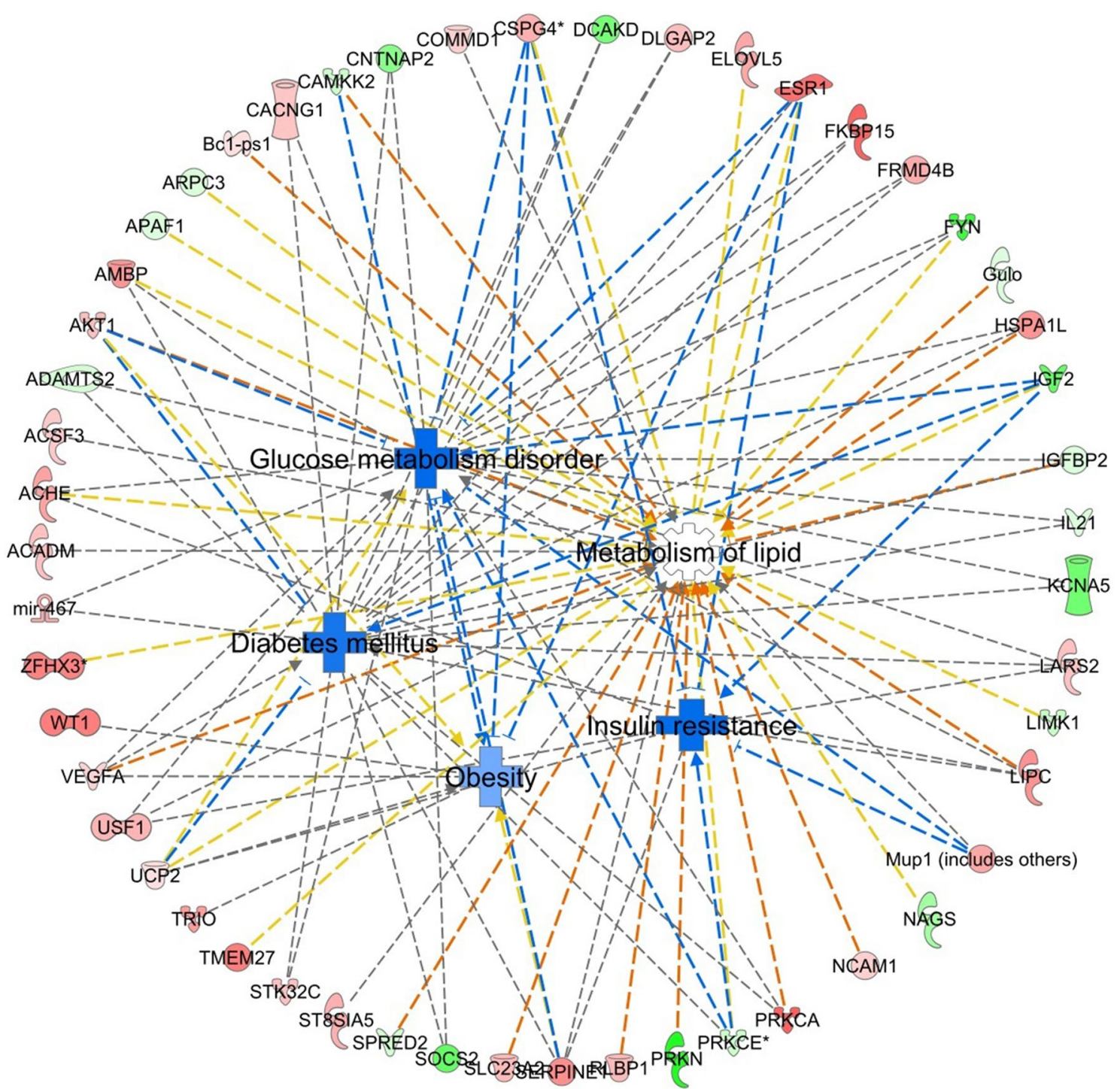

Fig. 3 IPA analysis of reduced representation bisulfite sequencing in D13.5 PGCs from F1-GDM male fetuses. DMGs enriched in glucose metabolism disorder, lipid metabolism disorder, obesity, insulin resistance, and diabetes mellitus. Network analysis was performed using Ingenuity Pathway Analysis. Red color indicates hypermethylation, green color indicates hypomethylation, a solid line indicates a direct interaction, a dashed line indicates an indirect interaction, and a line without an arrowhead indicates binding

methylation or mRNA expression significance of Fyn in liver tissues compared with control (Figs. 4f, 5b).

In muscle, the F2/H-GDM group exhibited lower overall methylation levels as well as at all five CpG sites of Fyn compared with control (Fig. 4g), and mRNA expression of Fyn was increased compared with the control group (Fig. 5c). In contrast, there were no differences in methylation or expression of Fyn between the F2/N-GDM and

(See figure on next page.)

Fig. 4 Targeted bisulfite pyrosequencing-based DNA methylation differences of Fyn in control, F1-, F2-, and F3-GDM male mice. a Methylation differences in D13.5 PGCs from control and GDM male fetuses ( $n=5$ pregnant mice per group). b Methylation differences in D18.5 control, F1and F2-GDM testes ( $n=8$ mice per group). c Methylation differences in sperm from control, F1/C-GDM, and F2/C-GDM mice at 8 weeks ( $n=5$ mice per group). $\mathbf{d}$ Methylation differences in D7.5 fetuses from control, F2-, and F3-GDM mice ( $n=5$ mice per group). e Methylation differences in adipose tissues from control, F2/H-GDM, and F2/N-GDM male mice at 8 weeks ( $n=5$ mice per group). $\mathbf{f}$ Methylation differences in liver tissues from control, F2/H-GDM, and F2/N-GDM male mice at 8 weeks ( $n=5$ mice per group). g Methylation differences in muscle tissues from control, F2/H-GDM, and F2/N-GDM male mice at 8 weeks ( $n=5$ mice per group). Data are presented as mean $\pm \mathrm{SEM} .{ }^{*} p<0.05,{ }^{* *} p<0.01,{ }^{* * *} p<0.001$ versus the corresponding control 


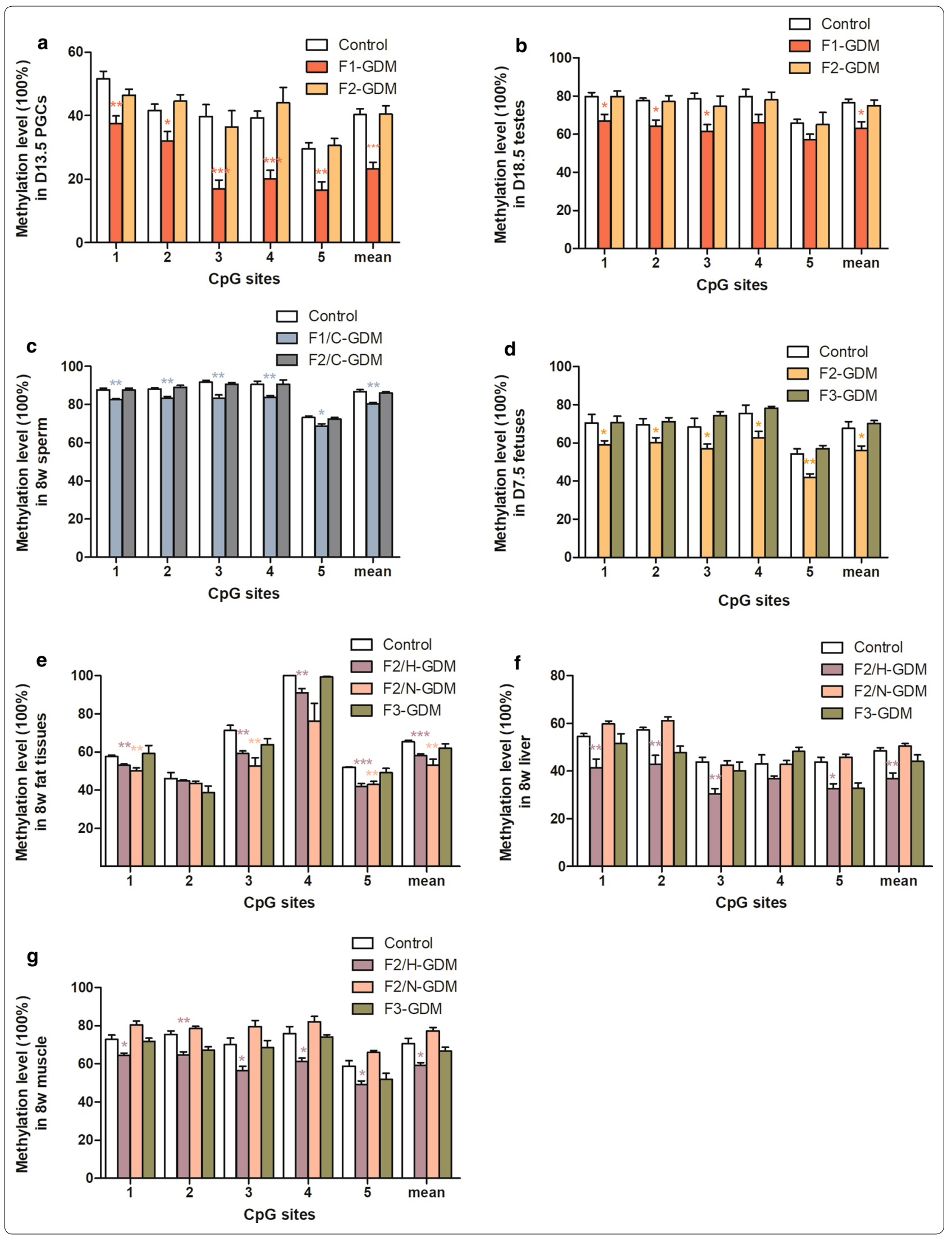




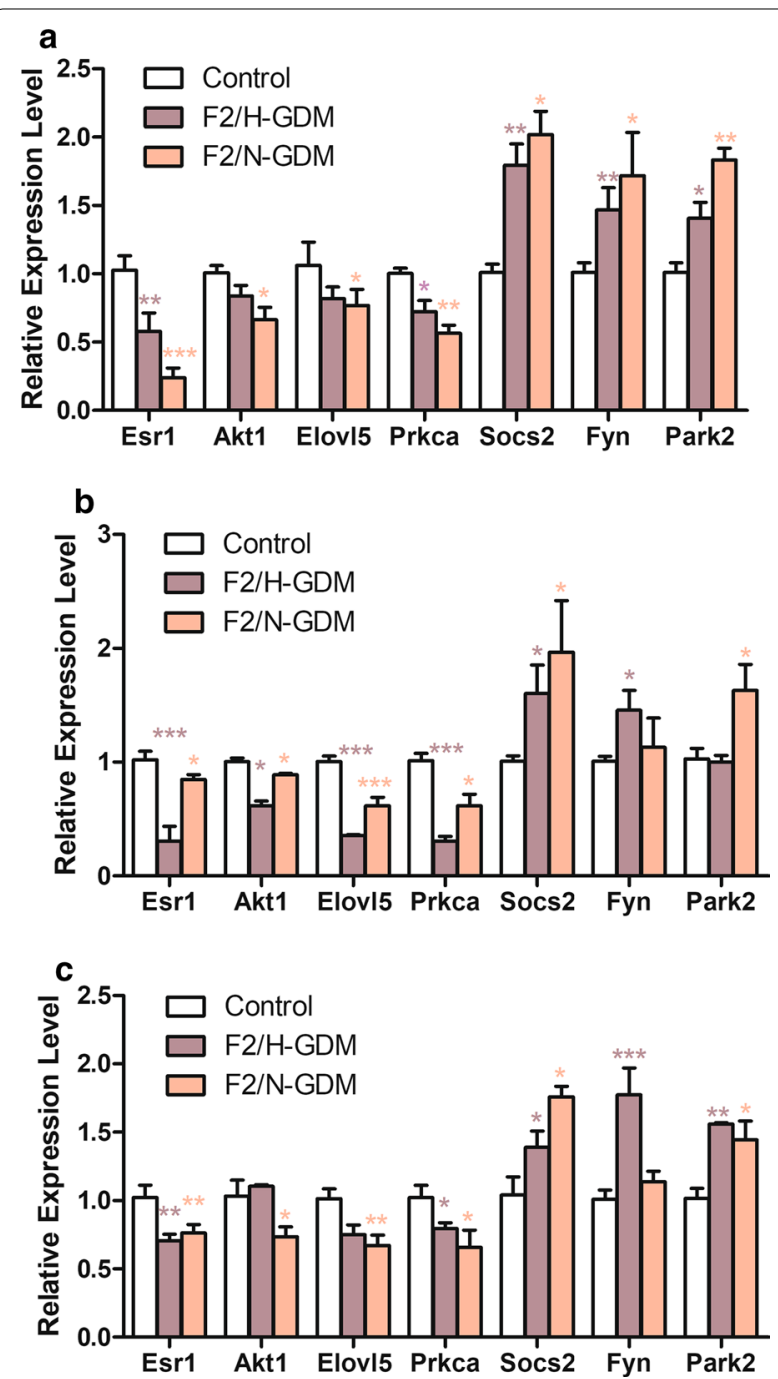

Fig. 5 Relative mRNA expression of targeted DMGs in control and F2-GDM mice. Gene expression levels in adipose (a), liver (b), and muscle (c) tissues from control, F2/H-GDM, and F2/N-GDM male mice at 8 weeks ( $n=5$ mice per group). Data are presented as mean \pm SEM. ${ }^{*} p<0.05,{ }^{* *} p<0.01,{ }^{* *} p<0.001$ versus the corresponding control

control groups (Figs. 4g, 5c). Methylation at these loci is likely to inhibit transcriptional activity, since low methylation levels are associated with high expression levels.

We finally measured Fyn methylation levels in D13.5 PGCs and D18.5 testes of F2-GDM, but found no difference between the F2-GDM and control groups (Fig. 4b). Sperm from F2/C-GDM mice consistently exhibited no methylation difference in Fyn when comparing with the control (Fig. 4c). Further, D7.5 F3-GDM fetuses also exhibited no methylation difference of Fyn when comparing with the control (Fig. 4d). Methylation assessment on germ cells of F2-GDM and fetus of F3-GDM provides epigenetic evidence that intrauterine hyperglycemia exposure might have no effect on F2 germ cells or F3 generation.

We also performed qPCR analysis of the other six candidate genes in the F2 generation to indirectly assess the relationship between the epigenetic reprogramming and corresponding gene expression. Four hypermethylated genes (Esr1, Akt1, Elovl5, and Prkca) and three hypomethylated genes (Socs2, Fyn, and Park2) from RRBS array were validated. The mRNA relative expression levels of these seven genes were examined in adipose (Fig. 5a), liver (Fig. 5b), and muscle (Fig. 5c) from male F2 generation mice. Compared with control, hypermethylated genes Esr1 and Prkca showed decreased mRNA expression in adipose, liver, and muscle of both $\mathrm{F} 2 / \mathrm{H}$ and F2/N-GDM groups; hypermethylated gene $A k t 1$ and Elovl5 showed decreased expression in all three tissues of F2/N-GDM mice and significantly decreased expression in liver of F2/H-GDM mice; hypomethylated gene Socs2 showed increased expression in adipose, liver, and muscle of both F2/H- and F2/N-GDM groups; hypomethylated gene Fyn showed increased expression in all three tissues of F2/H-GDM mice and significantly increased expression only in adipose of F2/N-GDM mice; hypomethylated gene Park2 showed increased expression in all three tissues of F2/N-GDM mice, and significantly increased expression only in adipose and muscle of F2/H-GDM mice. In general, the hypomethylated genes exhibited increased expression levels whereas the hypermethylated genes exhibited decreased expression (Fig. 5).

Together, these results suggest that the aberrant methylation caused by intrauterine hyperglycemia exposure in PGCs can be passed to fetal testes and mature sperm. In addition, this is a strong factor in determining the methylation status in somatic tissues but not germ cells of the F2 generation.

\section{Intrauterine hyperglycemia exposure had no effect on the $\mathrm{F} 3$ generation}

Based on the methylation assessment on F2-GDM germ cells and F3-GDM fetuses described above, the effects of intrauterine hyperglycemia exposure on the F3-GDM phenotypes were examined. F2-GDM mice without any abnormal phenotype were selected to produce F3-GDM progeny. At 8 weeks of age, there were no differences in GTT, ITT, bodyweight, HOMA-IR index, or serum biochemical parameters between F3-GDM and control mice (Fig. 6, Table 1). These results suggest that although intrauterine hyperglycemia exposure per se has a strong effect on the F2 generation, it has no effect on the F3 generation. This clarifies the role of intrauterine hyperglycemia exposure in intergenerational inheritance. 

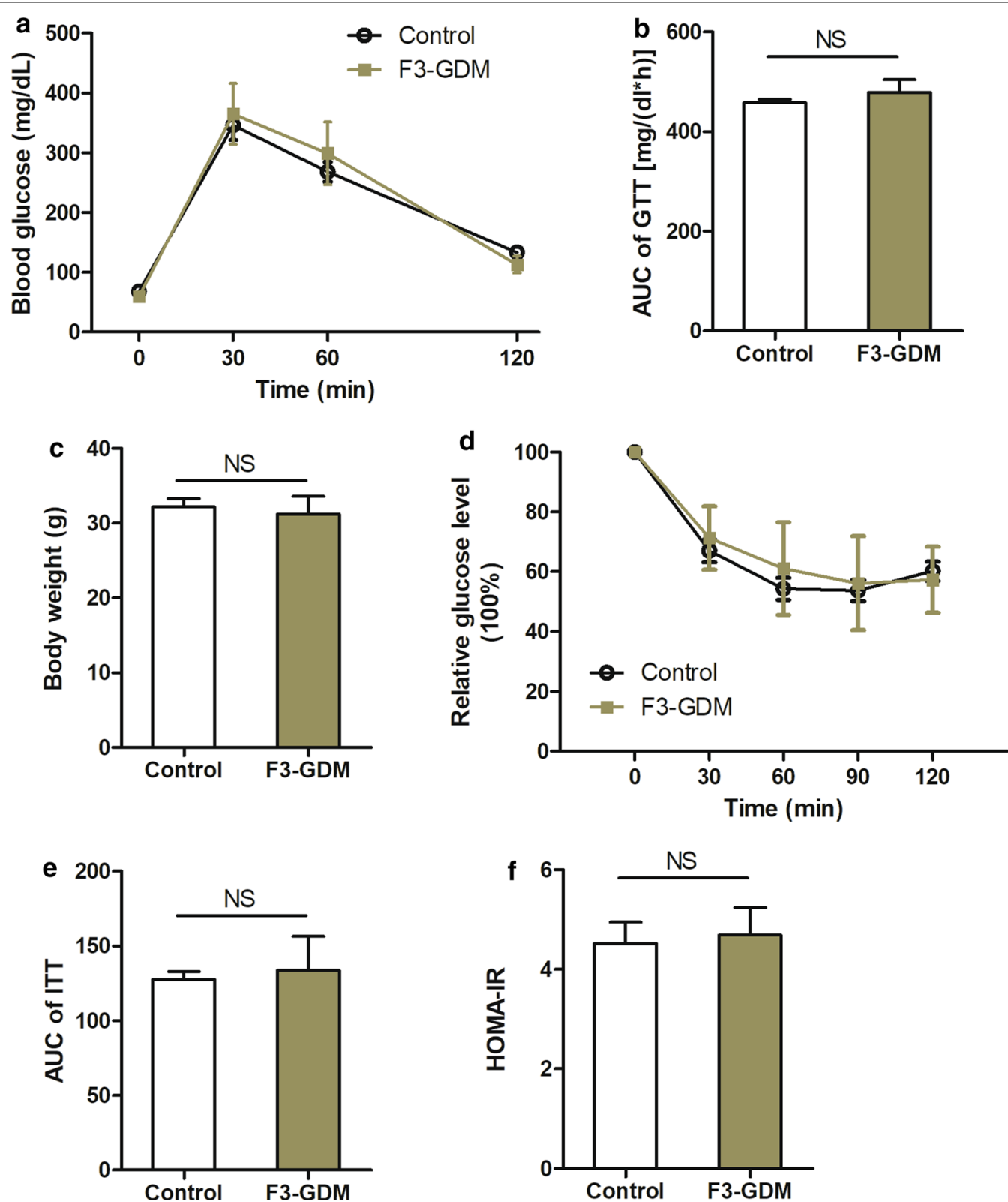

Fig. 6 F3-GDM mice demonstrated no metabolic change at adulthood. a GTT curve and $\mathbf{b}$ the resulting AUC of control and F3-GDM male mice at 8 weeks. c Bodyweight of control and F3-GDM male mice at 8 weeks. $\mathbf{d}$ ITT curve and $\mathbf{e}$ the resulting AUC of control and F3-GDM male mice at 8 weeks. f HOMA-IR index of control and F3-GDM male mice at 8 weeks. For each group in a-f, control $(n=10)$, F3-GDM $(n=10)$. Data are presented as mean $\pm \mathrm{SEM}$

\section{Discussion}

The present study demonstrated that intrauterine hyperglycemia affected the metabolic condition of the F2 generation but not the F3 generation and that the phenotype alteration of the F2 generation could be related to aberrant methylation erasure on day 13.5 PGCs of the
F1 generation. These results provide clear evidence for a gamete-based origin of adult disease and highlight the importance of distinguishing the intergenerational effect caused by in utero exposure and postnatal exposure. Schematic diagram of this study was presented as Additional file 7: Fig. S7. 
There were no significant differences in metabolic parameters between the F1/C-GDM male mice that were used as founders in the present study and control mice. Thus, we excluded adult influence factors, such as obesity, glucose metabolism dysfunction, and lipid metabolism dysfunction, which can be inheritable to progeny [19]. F2 male offspring of F1/C-GDM fathers exhibited impaired insulin sensitivity and increased body weight compared with control mice, and some F2 offspring developed impaired glucose tolerance at 8 weeks of age. These dysfunctions in the F2 offspring clearly demonstrated that intrauterine hyperglycemia exposure had a strong impact on grandchildren directly.

A previous study showed that the F2 offspring of F1-GDM fathers with IGT developed impaired glucose tolerance and decreased fasting insulin levels at 8 weeks, without alterations in bodyweight and fasting glucose levels [14]. The different metabolic features of the F2 generation and the $\mathrm{F} 1$ father indicate that both intrauterine exposure and postnatal metabolic exposure play a role in determining the acquired features of the progeny.

In the F1 generation, although F1-GDM mice exhibited glucose intolerance, none showed insulin resistance or increased bodyweight in the current study or previous reports [14]. Surprisingly, the F2 generation of healthy fathers had more diabetic phenotypes than the IGT F1-GDM mice in the current study. Thus, we speculate that intrauterine hyperglycemia may affect somatic cells and germ cells of the first generation differently, which could be caused by unsynchronized methylation reprogramming in somatic and germ cells [21]. In addition, this suggests that intrauterine exposure may have an impact on grandchildren well beyond direct effects on the F1 generation.

The current study revealed no metabolic changes in the F3-GDM generation born to healthy F2-GDM fathers. There was also no difference in the methylation status of Fyn in germ cells from F2/C-GDM mice or fetuses from the F3-GDM mice. These results suggest that aberrant methylation in gametes caused by intrauterine exposure can affect the F2 generation but is not heritable to the F3 generation.

Insulin resistance is a condition in which elevated insulin levels are required to maintain normal blood glucose. It is generally present in individuals with obesity and type II diabetes. Both genetic and environmental factors play a role in the development of insulin resistance [36-40]. Lipid accumulation is often coupled to an insulin resistant state [41]. When we compared the bodyweight of F1 and F2 generations of GDM, we supposed that abnormal lipid accumulation may be an important indicator of a severe metabolic dysfunction outcome in the F2 generation.
Taking the phenotype traits and IPA analysis together, we used Fyn as a candidate gene to study methylation and intergenerational inheritance in this study. Fyn is involved in the control of metabolism, inflammation, adipogenesis, and insulin signaling. Available data indicate a strong link between Fyn and the development of diabetes [34, 42]. In the current study, we observed consistent hypomethylation of Fyn from fetal PGCs to mature sperm in F1/C-GDM mice, then inherited by F2 somatic cells but not germ cells, and finally exhibited no methylation alteration in F3 generation. The methylation status of Fyn was synchronous with metabolic phenotypes presented in offspring, therefore providing a link between metabolic changes and epigenetic alteration in intergenerational transmission.

Overall, this study uniquely characterized intrauterine hyperglycemia exposure as an important determinant of diabetic outcome in F2 male offspring independent of postnatal environmental exposure, but not a weighting factor on metabolic and epigenetic changes in the F3 generation. In addition, intrauterine hyperglycemia exposure could induce aberrant methylation in PGCs as early as D13.5 during pregnancy.

It may be useful to track dynamic methylation status of from single embryo to adulthood individually. Unfortunately, up to date, there is no suitable way to do it. We hope that it could be performed in future.

\section{Conclusion}

Our data suggest that intrauterine hyperglycemia exposure alters DNA methylation patterns in D13.5 primordial germ cells of F1 generation, which could be maintained in somatic cells but not germ cells in F2 generation, thus causing an intergenerational inheritance of metabolic disorder in F2 but not F3 generation. Our results indicate the necessity of subdividing the exposure history occurred at different periods in life span and reconsider the origin of epigenetic and metabolic inheritance on F3 generation when studying $\mathrm{DOHaD}$.

\section{Additional files}

Additional file 1. Primers designed for pyrosequencing and $\mathrm{qPCR}$ in the study, and gender confirmation of D13.5 male PGCs.

Additional file 2. Metabolic phenotypes and bio-parameters of F2/CGDM male mice at 8 weeks.

Additional file 3. Distribution of differentially methylated loci in gene elements, and hierarchical clustering presentation of RRBS sequencing.

Additional file 4. Differentially methylated genes in D13.5 PGCs of F1-GDM mice.

Additional file 5. IPA pathway analysis of RRBS methylome sequencing. Additional file 6. DNA methylation validation in control and STZ-treated nondiabetic mice.

Additional file 7. Schematic diagram of the study 


\section{Abbreviations}

DOHaD: developmental origins of health and disease; GDM: gestational diabetes mellitus; PGCs: primordial germ cells; STZ: streptozotocin; GTT: glucose tolerance test; ITT: insulin tolerance test; AUC: area under the curve; TG: triglyceride; TC: total cholesterol; HDL: high-density lipoprotein; LDL: low-density lipoprotein; HOMA-IR: homeostasis model of insulin resistance; RRBS: reduced representation bisulfite sequencing; DML: differentially methylated loci; DMRs: differentially methylated regions; IPA: Ingenuity Pathway Analysis.

\section{Authors' contributions}

$\mathrm{JR}, \mathrm{HFH}$, and JZS designed the experiments; JR and YC performed the animal experiments and pyrosequencing and collected and analyzed RRBS data; ZHM and XYD performed the animal experiments; $Y Z Z$ and GLD performed $q P C R$ experiments; HYP, TUR, and RA collected animal materials; and JR, HFH, and JZS wrote and edited the manuscript. All authors read and approved the final manuscript.

\section{Authors' information}

Hefeng Huang M.D., is an academician of Chinese Academy of Sciences; professor of obstetrics and gynecology; president of the International Peace Maternity and Child Health Hospital, School of Medicine, Shanghai Jiao Tong University, China. Jianzhong Sheng M.D., Ph.D., is a professor of Department of Pathology \& Pathophysiology, School of Medicine, Zhejiang University, China. Both professors focus on gametes and fetal origins of adult diseases and were invited by Springer publishing book "Gamete and Embryo-fetal Origins of Adult Diseases."

\section{Author details}

'Department of Pathology and Pathophysiology, School of Medicine, Zhejiang University, Hangzhou, China. ${ }^{2}$ The Key Laboratory of Reproductive Genetics (Zhejiang University), Ministry of Education, Hangzhou, China. ${ }^{3}$ Department of Reproductive Endocrinology, Zhejiang Women's Hospital, School of Medicine, Zhejiang University, Hangzhou, China. ${ }^{4}$ The International Peace Maternity and Child Health Hospital, School of Medicine, Shanghai Jiao Tong University, Shanghai, China.

\section{Acknowledgements}

Jian-Zhong Sheng is the first (major)corresponding author and He-Feng Huang is the second corresponding author.

\section{Competing interests}

The authors declare that they have no competing interests.

\section{Availability of data and materials}

The RRBS data reported in this paper have been deposited in the NCBI Gene Expression Omnibus (GEO) database with accession number GSE108319. Supplemental data were attached as additional files. More data requested were available from the corresponding author on reasonable request.

\section{Consent for publication}

All authors have approved the manuscript and agree with its submission to Epigenetics \& Chromatin

\section{Ethics approval and consent to participate}

No human materials were included in the study. The animal ethics and protocol were approved by the Zhejiang University Institutional Animal Care and Use Committee.

\section{Funding}

This study was supported by the NSFC-CIHR Joint Health Research Program (No. 8161101434 to H.F.H.), the National Natural Science Foundation of China (No. 31671569 to J.Z.S., No. 81490742 and 31471405 to H.F.H), the NSFC-CIHR Joint Health Research Programs (81361128007 to J.-Z.S.), and a Canadian Institutes for Health Research China-Canada Joint Health Research Initiative grant (CCI-132570 to P.C.K.L.).

\section{Publisher's Note}

Springer Nature remains neutral with regard to jurisdictional claims in published maps and institutional affiliations.
Received: 8 February 2018 Accepted: 21 May 2018

Published online: 25 May 2018

\section{References}

1. Adair L. Developmental origins of health and disease. Science. 2007;315(5812):600-1.

2. Li J, Tsuprykov O, Yang XP, Hocher B. Paternal programming of offspring cardiometabolic diseases in later life. J Hypertens. 2016;34(11):2111-26.

3. Wolstenholme JT, Edwards M, Shetty SRJ, Gatewood JD, Taylor JA Rissman EF, Connelly JJ. Gestational exposure to Bisphenol A produces transgenerational changes in behaviors and gene expression. Endocrinology. 2012;153(8):3828-38

4. Laubenthal J, Zlobinskaya O, Poterlowicz K, Baumgartner A, Gdula MR, Fthenou E, Keramarou M, Hepworth SJ, Kleinjans JC, van Schooten FJ, Brunborg G, Godschalk RW, Schmid TE, Anderson D. Cigarette smokeinduced transgenerational alterations in genome stability in cord blood of human F1 offspring. FASEB J Off Publ Fed Am Soc Exp Biol. 2012:26(10):3946-56.

5. Luo H, Deng Z, Liu L, Shen L, Kou H, He Z, Ping J, Xu D, Ma L, Chen L, Wang $\mathrm{H}$. Prenatal caffeine ingestion induces transgenerational neuroendocrine metabolic programming alteration in second generation rats. Toxicol Appl Pharmacol. 2014:274(3):383-92.

6. Fujii T. Transgenerational effects of maternal exposure to chemicals on the functional development of the brain in the offspring. Cancer Causes Control. 1997:8(3):524-8.

7. Huang HF, Sheng JZ. Gamete and embryo-fetal origins of adult diseases. Amsterdam: Springer; 2014

8. Buchanan TA, Xiang AH, Page KA. Gestational diabetes mellitus: risks and management during and after pregnancy. Nat Rev Endocrinol. 2012;8(11):639-49.

9. Reece EA, Leguizamon G, Wiznitzer A. Gestational diabetes: the need for a common ground. Lancet. 2009;373(9677):1789-97.

10. Aris IM, Soh SE, Tint MT, Liang S, Chinnadurai A, Saw SM, Rajadurai VS, Kwek K, Meaney MJ, Godfrey KM, Gluckman PD, Yap FK, Chong YS, Lee YS. Effect of maternal glycemia on neonatal adiposity in a multiethnic Asian birth cohort. J Clin Endocrinol Metab. 2014;99(1):240-7.

11. Quilter CR, Cooper WN, Cliffe KM, Skinner BM, Prentice PM, Nelson L, Bauer J, Ong KK, Constancia M, Lowe WL, Affara NA, Dunger DB. Impact on offspring methylation patterns of maternal gestational diabetes mellitus and intrauterine growth restraint suggest common genes and pathways linked to subsequent type 2 diabetes risk. FASEB J 2014:28(11):4868-79.

12. Landon MB, Rice MM, Varner MW, Casey BM, Reddy UM, Wapner RJ, Rouse DJ, Biggio JR Jr, Thorp JM, Chien EK, Saade G, Peaceman AM, Blackwell SC, VanDorsten JP, H. Eunice Kennedy Shriver National Institute of Child, N. Human Development Maternal-Fetal Medicine Units. Mild gestational diabetes mellitus and long-term child health. Diabetes Care. 2015:38(3):445-52.

13. Pereira TJ, Fonseca MA, Campbell KE, Moyce BL, Cole LK, Hatch GM Doucette CA, Klein J, Aliani M, Dolinsky VW. Maternal obesity characterized by gestational diabetes increases the susceptibility of rat offspring to hepatic steatosis via a disrupted liver metabolome. J Physiol. 2015:593(14):3181-97.

14. Ding GL, Wang FF, Shu J, Tian S, Jiang Y, Zhang D, Wang N, Luo Q, Zhang $Y$, Jin F, Leung PCK, Sheng JZ, Huang HF. Transgenerational glucose intolerance with Igf2/H19 epigenetic alterations in mouse islet induced by intrauterine hyperglycemia. Diabetes. 2012;61(5):1133-42.

15. Wei YC, Yang CR, Wei YP, Zhao ZA, Hou Y, Schatten H, Sun QY. Paternally induced transgenerational inheritance of susceptibility to diabetes in mammals. Proc Natl Acad Sci USA. 2014;111(5):1873-8.

16. Heard E, Martienssen RA. Transgenerational epigenetic inheritance: myths and mechanisms. Cell. 2014:157(1):95-109.

17. Quilter CR, Cooper WN, Cliffe KM, Skinner BM, Prentice PM, Nelson L, Bauer J, Ong KK, Constancia M, Lowe WL, Affara NA, Dunger DB. Impact on offspring methylation patterns of maternal gestational diabetes mellitus and intrauterine growth restraint suggest common genes and pathways linked to subsequent type 2 diabetes risk. FASEB J Off Publ Fed Am Soc Exp Biol. 2014:28(11):4868-79. 
18. Lambrot R, Xu C, Saint-Phar S, Chountalos G, Cohen T, Paquet M, Suderman M, Hallett M, Kimmins S. Low paternal dietary folate alters the mouse sperm epigenome and is associated with negative pregnancy outcomes. Nat Commun 2013;4:2889.

19. Ng SF, Lin RC, Laybutt DR, Barres R, Owens JA, Morris MJ. Chronic high-fat diet in fathers programs beta-cell dysfunction in female rat offspring. Nature. 2010;467(7318):963-6.

20. Carone BR, Fauquier L, Habib N, Shea JM, Hart CE, Li R, Bock C, Li C, Gu H, Zamore PD, Meissner A, Weng Z, Hofmann HA, Friedman N, Rando OJ. Paternally induced transgenerational environmental reprogramming of metabolic gene expression in mammals. Cell. 2010;143(7):1084-96.

21. Saitou M, Kagiwada S, Kurimoto K. Epigenetic reprogramming in mouse pre-implantation development and primordial germ cells. Development. 2012;139(1):15-31.

22. ReikW, Walter J. Genomic imprinting: parental influence on the genome. Nat Rev Genet. 2001;2(1):21-32.

23. Hajkova P, Erhardt S, Lane N, HaafT, El-Maarri O, Reik W, Walter J, Surani MA. Epigenetic reprogramming in mouse primordial germ cells. Mech Dev. 2002;117(1-2):15-23.

24. Kafri T, Ariel M, Brandeis M, Shemer R, Urven L, McCarrey J, Cedar H, Razin A. Developmental pattern of gene-specific DNA methylation in the mouse embryo and germ line. Genes Dev. 1992;6(5):705-14.

25. Harris IS, Treskov I, Rowley MW, Heximer S, Kaltenbronn K, Finck BN, Gross RW, Kelly DP, Blumer KJ, Muslin AJ. G-protein signaling participates in the development of diabetic cardiomyopathy. Diabetes. 2004;53(12):3082-90

26. Kim SJ, Nian C, Doudet DJ, McIntosh CH. Inhibition of dipeptidy I peptidase IV with sitagliptin (MK0431) prolongs islet graft survival in streptozotocin-induced diabetic mice. Diabetes. 2008;57(5):1331-9.

27. Feng $\mathrm{H}$, Conneely KN, Wu H. A Bayesian hierarchical model to detect differentially methylated loci from single nucleotide resolution sequencing data. Nucleic Acids Res. 2014;42(8):e69.

28. Hajkova P, Erhardt S, Lane N, Haaf T, El-Maarri O, Reik W, Walter J, Surani MA. Epigenetic reprogramming in mouse primordial germ cells. Mech Dev. 2002;117(1-2):15-23.

29. Hers I, Vincent EE, Tavare JM. Akt signalling in health and disease. Cell Signal. 2011;23(10):1515-27.

30. Tripathy S, Torres-Gonzalez M, Jump DB. Elevated hepatic fatty acid elongase- 5 activity corrects dietary fat-induced hyperglycemia in obese C57BL/6J mice. J Lipid Res. 2010;51 (9):2642-54.

31. Ribas V, Nguyen MT, Henstridge DC, Nguyen AK, Beaven SW, Watt MJ Hevener AL. Impaired oxidative metabolism and inflammation are associated with insulin resistance in ERalpha-deficient mice. Am J Physiol Endocrinol Metab. 2010;298(2):E304-19.

32. Miele C, Riboulet A, Maitan MA, Oriente F, Romano C, Formisano $P$, Giudicelli J, Beguinot F, Van Obberghen E. Human glycated albumin affects glucose metabolism in L6 skeletal muscle cells by impairing insulin-induced insulin receptor substrate (IRS) signaling through a protein kinase $\mathrm{C}$ alpha-mediated mechanism. J Biol Chem. 2003;278(48):47376-87.

33. Zadjali F, Santana-Farre R, Vesterlund M, Carow B, Mirecki-Garrido M, Hernandez-Hernandez I, Flodstrom-Tullberg M, Parini P, Rottenberg M Norstedt G, Fernandez-Perez L, Flores-Morales A. SOCS2 deletion protects against hepatic steatosis but worsens insulin resistance in high-fat-dietfed mice. FASEB J Off Publ Fed Am Soc Exp Biol. 2012;26(8):3282-91.

34. Sun Y, Ma YC, Huang J, Chen KY, McGarrigle DK, Huang XY. Requirement of SRC-family tyrosine kinases in fat accumulation. Biochemistry. 2005:44(44):14455-62.

35. Martin-Maestro P, Gargini R, Perry G, Avila J, Garcia-Escudero V. PARK2 enhancement is able to compensate mitophagy alterations found in sporadic Alzheimer's disease. Hum Mol Genet. 2016;25(4):792-806.

36. Huypens P, Sass S, Wu M, Dyckhoff D, Tschop M, Theis F, Marschall S, Hrabe de Angelis M, Beckers J. Epigenetic germline inheritance of dietinduced obesity and insulin resistance. Nat Genet. 2016;48(5):497-9.

37. Roberts-Toler C, O'Neill BT, Cypess AM. Diet-induced obesity causes insulin resistance in mouse brown adipose tissue. Obesity. 2015:23(9):1765-70

38. Caprio S. Insulin resistance in childhood obesity. J Pediatric Endocrinol Metab. 2002;15(Suppl 1):487-92.

39. Eckel RH, Grundy SM, Zimmet PZ. The metabolic syndrome. Lancet. 2005:365(9468):1415-28.

40. Thota P, Perez-Lopez FR, Benites-Zapata VA, Pasupuleti V, Hernandez AV. Obesity-related insulin resistance in adolescents: a systematic review and meta-analysis of observational studies. Gynecol Endocrinol 2017;33(3):179-184.

41. Shulman Gl. Cellular mechanisms of insulin resistance. J Clin Investig. 2000;106(2):171-6.

42. Bastie CC, Zong H, Xu J, Busa B, Judex S, Kurland IJ, Pessin JE. Integrative metabolic regulation of peripheral tissue fatty acid oxidation by the SRC kinase family member Fyn. Cell Metab. 2007;5(5):371-81.
Ready to submit your research? Choose BMC and benefit from:

- fast, convenient online submission

- thorough peer review by experienced researchers in your field

- rapid publication on acceptance

- support for research data, including large and complex data types

- gold Open Access which fosters wider collaboration and increased citations

- maximum visibility for your research: over $100 \mathrm{M}$ website views per year

At $\mathrm{BMC}$, research is always in progress.

Learn more biomedcentral.com/submissions 